\title{
Rare conditions of gastrointestinal bleeding
}

\author{
Hrbaty B, Reis R, Cambal M, Sekac J, Kostka V, Labas P, Vician M, Skultety J \\ 1st Department of Surgery, Faculty of Medicine, Comenius University, Bratislava, Slovakia. \\ boris.hrbaty@pobox.sk
}

\begin{abstract}
The aim of this paper is to analyze and discuss an infrequent topic, i. e. rare conditions of gastrointestinal bleeding.

Bleeding from gastrointestinal system is a common cause of acute abdomen and the most frequent causes are generally known.

Clinicians, first of all emergency medicine staff, surgeons and internists (gastroenterology specialists), general practitioners, or less frequently other specialists, sometimes meet with less common causes of acute or chronic bleeding from gastrointestinal organs. It is quite important to be aware of the possibility the bleeding being caused by some rare condition, which is not so often met in medical practice, because of their great diagnostic and therapeutic problem.

The low index of expectation of such a rare etiological unit could be the reason why destiny of such patients can be extremely problematic and why clinicians may fail at management of these patients (Fig. 4, Ref. 8). Full Text in PDF www.elis.sk.

Key words: gastrointestinal bleeding, acute abdomen, chronic bleeding.
\end{abstract}

Gastrointestinal bleeding is a frequent problem met in general practice. From the view of financial costs it represents a great portion of money given to health care in each society. Diagnostics and therapy of these patients can be very expensive. Acute bleeding from upper part of gastrointestinal system belongs to most frequent urgent situations in medical practice (Jurgoš et al, 2006).

Gastrointestinal bleeding is classified according to localization to bleeding from upper and lower gastrointestinal tract (the border line creates "ligamentum Treitzi”), according to intensity to acute and chronic bleeding. This classification does not require any comment.

Also the most frequent causes of gastrointestinal hemorrhage are generally known:

esophageal varices, erosions and ulcers of gastroduodenum, syndrome Mallory - Weiss, inflammations and tumors of gullet, stomach, duodenum; ulcers in gastrointestinal anastomoses, vessel fistulas (entero - vascular fistulas), affections of anorectum (hemorrhoids, fissures, proctitis), diverticles, inflammations and tumors of intestine (IBD, ischemic colitis, polyps, enterocolitis, carcinoma of large intestine, postirradiative colitis) (Jurgoš et al, 2006).

It is true, that these etiological units of a complex set of gastrointestinal bleedings mean large costs for medical care; but they do not represent neither diagnostic, nor curative problem. Because of their high frequency of occurrence, and accessibility to diagnostic procedures, (especially endoscopy and angiography),

1st Department of Surgery, Faculty of Medicine, Comenius University, Bratislava, Slovakia

Address for correspondence: B. Hrbaty, MD, 1st Department of Surgery, Faculty of Medicine, Comenius University, Mickiewiczova 13, SK-813 69 Bratislava, Slovakia. is their therapy and diagnostics standard and only exceptionally mean problem for managing them.

Nevertheless the situation is quite different, if the cause of gastrointestinal bleeding is less frequent, or rare etiological unit, which is not expected in common medical practice.

It can even be said, that most of us (i.e. physicians) never met such rare cause of gastrointestinal bleeding.

These rare etiological units include:

- Ehlers-Danlos syndrome (type IV),

- pseudoxanthoma elasticum,

- Marfan syndrome,

- very rarely some other heritable disorders of connective tissue, as:

- osteogenesis imperfecta,

- cutis laxa,

- Wener syndrome,

- Menkes syndrome (Beylot et al, 1994),

- hemoptysis,

- nasopharyngeal bleeding and hemorrhage in the case of esophageal rupture,

- hemophilia,

- angiodysplasias,

- amyloidosis,

- lesion Dieulafoy (Jurgoš et al, 2006).

Arterial involvement in most of these diseases is connected with unusual bleeding, especially from gastrointestinal organs. It is not only an important feature of the diagnosis, but also of prognosis of these disorders of connective tissue (Beylot et al, 1994).

The first three diseases mentioned in the classification, i.e.: Syndrome Ehlers-Danlos, Marfan Syndrome and Pseudoxanthoma elasticum, are probably the "most frequent" among these rare 

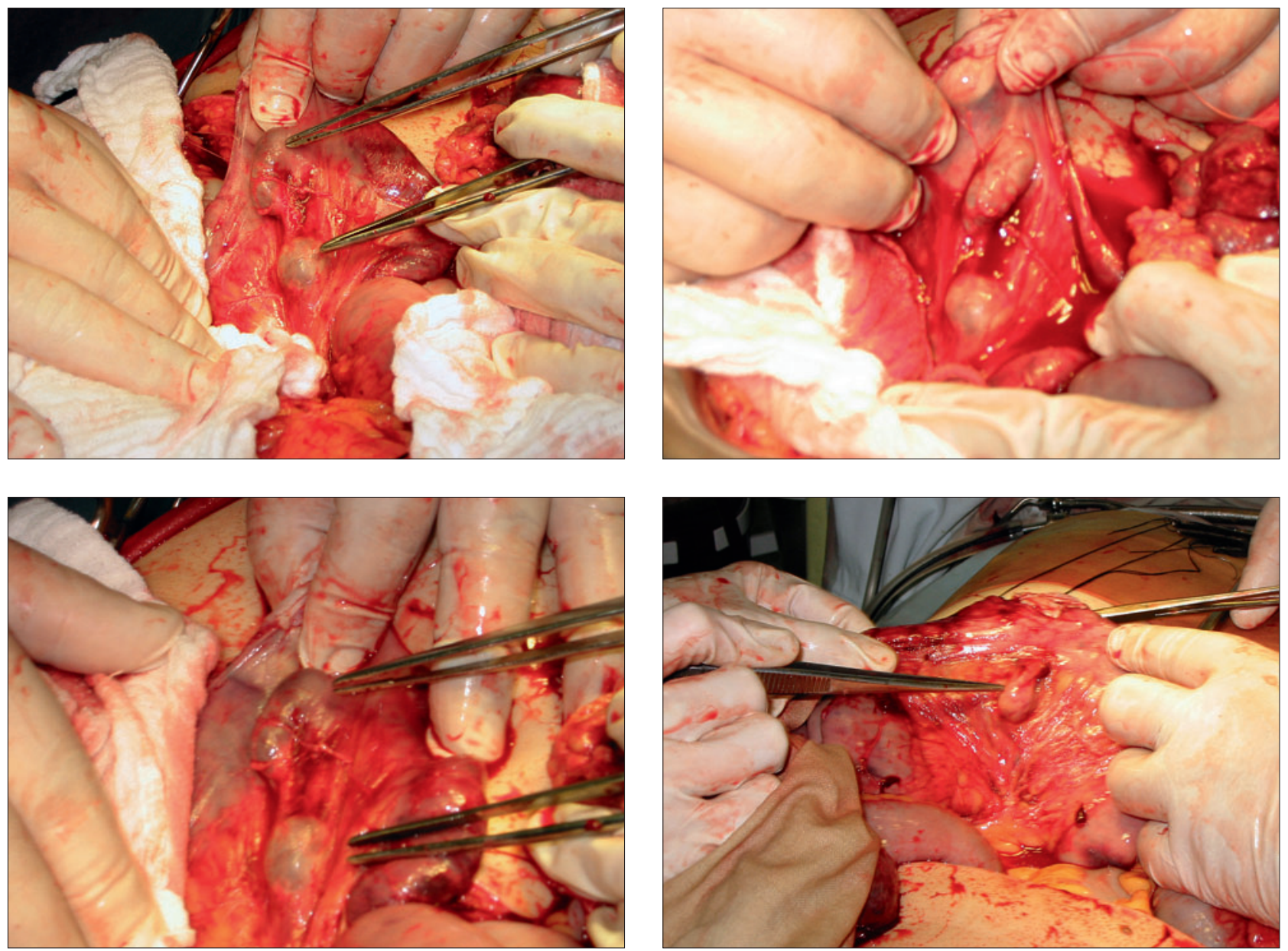

Figures 1-4. Figures from the operation, depicting the shape and localization of aneurysms. The surgical instruments are pointing at aneurysms in the course of jejunal artery.

conditions of gastrointestinal bleeding and the first one of them (EDS) constitutes the main area of this paper.

Ehlers-Danlos syndrome is a heterogenous group of connective tissue disorders, consisting of nine main types. Type IV of this syndrome (synonym: Sack-Barabas syndrome, Ehlers-Danlos arterial syndrome), is characterized by unexpected spontaneous rupture of larger arteries, formation of aneurysms and recurrent spontaneous haemathomas. Death occurs usually after aortic rupture or rupture of another large artery (Lazovskis, 1990).

The severity of Marfan Syndrome is due to aortic involvement. Aneurysm of aorta (usually ascending aorta) represents a vital risk of rupture. Serious threat means also aortic dissection.

In Pseudoxanthoma elasticum is a progressive occlusive syndrome associated with bleeding, again especially from gastrointestinal organs (Beylot et al, 1994).

\section{Material and methods}

The aim of this paper is to present our experience with diagnostics and therapy of a patient with severe acute gastrointestinal bleeding caused by Ehlers-Danlos syndrome type IV.
We have to remark, that the diagnostics of EDS is frequently complicated. The phenotypical variance that characterizes this syndrome usually makes its recognition rather difficult and failure in diagnostics is not uncommon (Solomon et al, 1996).

The diagnosis of EDS - type IV in the case of our patient was known and done years before the lethal episode of catastrophic gastrointestinal bleeding.

Results - Case report of a patient with massive gastrointestinal bleeding caused by Ehlers-Danlos syndrome type IV arterial

25 years old patient was admitted to 1st Department of Surgery, University Hospital of Comenius University, Mickiewiczova Street, Bratislava, Slovak Republic, with intermittent gastrointestinal hemorrhage, to perform panendoscopic investigation of the whole GUT to determine the source of this bleeding, not specified, neither with gastroscopy, nor with coloscopy (Figs 1-4).

From his personal history was known, that in 1983 splenectomy was performed due to portal hypertension. In 1984 operation for scrotal hernia on both sides was performed. Between the 
years 1994 - 1999, this patient was ten times admitted to regional hospital with the diagnosis of bleeding esophageal varices with recurrent sclerotherapy. In 1999 neurological difficulties occurred in the sense of consciousness disorders, epilepsy paroxysms and suspicion of stroke and on their basis panangiographic investigation was performed, finding multiple aneurysms of cerebral vessels. This status was operated at neurosurgery department with clipping the necks of aneurysms.

In the period of neurosurgical operation was in the course of 1999 the diagnosis of Ehlers-Danlos syndrome type IV arteriale definitely established by genetic investigation.

In 2003 was the patient admitted to regional hospital with 2 days history of enterorrhagia. Gastroscopy and coloscopy both did not succeed in finding the source of hemorrhage. Although given the adequate hemostyptic therapy and hemosubstitution, the bleeding continued. Transfer to greater hospital was performed to finish the diagnostic procedure. But selective angiography also did not reveal the source of bleeding. Another transfer to University Hospital was done to perform panendoscopic investigation of GUT to identify the source of hemorrhage. The bleeding continued with small intensity. But in the day of endoscopy, before it could be finished, acute massive gastrointestinal bleeding forced the surgical staff to perform acute laparotomy. The before performed splenectomy was the cause of massive intraabdominal adhesions, which made the revision of GUT very complicated. After identification of the whole of small intestine, this was revised for the source of bleeding. Cirrhosis and ascites were present. An enterotomy approximately $40 \mathrm{~cm}$ aborally from Treitz was done and intestinoscopy orally and aborally performed, but the large amount of blood disabled to reveal the source of bleeding. Suction of intestinal content was necessary, and another intestinocopy revealed the source of bleeding approximately $20 \mathrm{~cm}$ aborally from Treitz ligament. Aneurysmatic dilatation of jejunal artery was identified in mesentery - in the course of this artery three aneurysmatic sacs were present (see fotodocumentation) and after enterotomy in this region the surgeon revealed an active hemorrhage from aneurysm opening to intestinal lumen.

The jejunal artery was carefully identified to its healthy segment, ligated and resection of adjacent $15 \mathrm{~cm}$ segment of proximal jejunum with pathologically changed vessels was performed.

Unfortunately, 29 hours after laparotomy, the patients dies due to multiorgan failure as a consequence of protrahent haemorrhagic shock .

\section{Discussion}

Diagnosis of EDS is paramount, however life - threatening associations have to be searched for and unique principles of management can be instituted. Patients with EDS are prone to gastrointestinal catastrophes such as perforation and massive bleeding, which can be compounded by grave surgical and vascular complications. A thorough knowledge of the gastrointestinal manifestations of EDS and their management is mandatory to prevent unnecessary morbidity and mortality (Solomon et al, 1996).

EDS type IV presents a diagnostic and therapeutic challenge to the primary care physician, surgeon, and rheumatologist. In patients for whom the diagnosis is known, avoidance of trauma, contact sports, or strenuous activities, joint bracing and protection, and counseling on contraception are helpful preventive strategies. In patients presenting with vascular, gastrointestinal, or obstetric complications, a history of hypermobility and skin fragility should lead to a suspicion of this diagnosis, and to caution in the use of certain invasive diagnostic and operative techniques. Efforts should be made to examine family members. Most importantly, when caring for such patients, the acute onset of headaches, chest pain, shortness of breath, and abdominal pain should arouse suspicion of a potentially catastrophic vascular or visceral event. (SantosOcampo et al, 1999).

This type of EDS (or so called arterial - ecchymotic type) is of concern to vascular surgeons because it is frequently associated with spontaneous catastrophic bleeding. Patients with EDS type IV present with easy „bruisability“, hypermobility of their joints, transparent skin and excessive elasticity of the skin. These patients are prone to episodes of acute hemorrhage, and necessity of vascular surgical procedures. Very frequent are aneurysms or a dissection, arteriovenous fistulas. Arteriography at these patients can be associated with a complication rate up till $67 \%$. The prognosis for them is poor; up till $44 \%$ die before surgery and $19 \%$ die during the operative period. (Cikrit et al, 1987).

Bleeding should be managed nonoperatively when possible. $51 \%$ of these patients die before reaching 40 years of age. Arteriography should be avoided. The standard repair of aneurysms and perforations is unlikely to be successful because of marked vessel friability. Bleeding vessels should be repaired with buttressed sutures and no tension or should be ligated. Genetic counseling with assay of collagen production is recommended for family members. (Cikrit et al, 1987).

In the recent years more optimistic works were published:

There has been a debate regarding the safety of performing elective procedures in patients with vascular manifestations associated with Ehlers-Danlos syndrome. The elective surgical management of vascular disorders in EDS patients using open and endovascular procedures has been associated with good outcomes. Results of these works suggest that vascular interventions in these EDS patients can be safely performed and should not be withheld until rupture or acute symptoms arise. (Brooke et al, 2010).

Pregnant women with the vascular type of EDS should be followed in a high-risk obstetrical program. Affected individuals should be instructed to seek immediate medical attention for sudden unexplained pain. (Pepin et al, 2006).

\section{Conclusion}

Detection of the bleeding sources localized in the esophagus, colon, stomach or duodenum does not seem to be too difficult under standard circumstances. Much more problematic is the search for the cause of bleeding, which is located in the small intestine. In about $1-10 \%$ the attempts to ascertain the source of bleeding are failing even by repeated endoscopic examination of the proximal or distal part of the GUT (GFS vs CFS). It is in these cases when it is necessary to try to find the 
cause of bleeding in the small intestine (Jurgoš et al, 2006). If problems with identifying the source of gastrointestinal bleeding persist even by exhausting all available options (or in case of acute episode with necessity of emergent laparotomy) we deem as appropriate operational revision with peroperative endoscopy, for the purpose of locating the source of haemorrhage. We believe, based on our experience, that it is appropriate to indicate operational revision in earlier stages before developing irreversible changes or before excessive ammount of blood transfusions are given, when even identification and elimination of the source of bleeding are not able to save patient's life. Either way even in the case of a timely and successful operation for such an acute vascular - haemorrhagic stroke, patients with vascular type of EDS come to live on average for 30-35 years and die in an attack similar to presented case.

\section{References}

1. Beylot C, Doutre MS, Beylot-Barry M, Busquet M. Arterial involvement in hereditary dysplasia of the connective tissue. Rev Med Internal 1994; 15 (3): 193-209.
2. Brooke BS, Arnaoutakis G, Mc Donnell NB, Black JH: Contemporary management of vascular complications associated with Ehlers-Danlos syndrome. J Vasc Surg 2010; 51 (1): 131-138; discussion 138-139.

3. Cikrit DF, Miles JH, Silver D. Spontaneous arterial perforation: the Ehlers-Danlos specter. J Vasc Surg 1987; 9 (2): 248-255.

4. Jurgoš L et al. Gastroenterológia. Bratislava, 2006, pp 211.

5. Lazovskis J. Přehled klinických symptomů a syndromů. Praha: Avicenum, 1990, 581 pp.

6. Pepin MG, Byers PH. Ehlers-Danlos syndrome, Vascular Type. In: Pagon RA, Bird TC, Dolan CR, Stephens K (Eds). GeneReviews (Internet). Seattle (WA): University of Washington, Seattle; 1993-1999 Sep 02 (updated 2006 Jun 07).

7. Santos-Ocampo AS, Hoffman GS. Aneurysms and hypermobility in a 45-year-old woman. Clev Clin J Med 1999; 66 (7): 426-433.

8. Solomon JA, Abrams L, Lichtenstein GR. Gastrointestinal manifestations of Ehlers - Danlos syndrome. Amer J Gastroenterol 1996; 91 (11): 2282-2288.

Received October 10, 2010. Accepted January 9, 2012. 